\title{
用参照设计刻画最小附加混杂 部分因析裂区设计*
}

\author{
艾明要 ${ }^{* *}$ \\ (北京大学数学科学学院数学与应用数学实验室, 北京 100871) \\ 张润楚 \\ (南开大学数学科学学院核心数学与组合数学重点实验室, 天津 300071)

\begin{abstract}
摘要用参照设计刻画部分因析设计的特征对于构造近似饱和的因析设计是 十分有效的, 因为此时的参照设计很小. Mukerjee 和方开泰利用投影几何理论将 一个正规对称部分因析裂区 (FFSP) 设计的附加字长型用其补子集表示出来，然 而其表示形式并不统一。基于因析设计理论和编码理论之间的联系，得到了联 系一个正规对称或混合水平 FFSP 设计与其参照设计的附加字长型之间关系的一 般而统一的组合等式, 根据这些等式进一步建立了通过参照设计来识别最小附 加混杂对称或混合水平 FFSP 设计的一般而统一的规则.
\end{abstract}

\section{关键词 编码理论 参照设计 最小附加混杂 部分因析裂区设计 投影几何 字长型}

\section{1 引言}

部分因析 (FF) 设计已经广泛应用于工业和农业实验中. 由 Fries 和 Hunter ${ }^{[1]}$ 提出的最小低阶混杂 (MA) 准则普遍用于筛选好的正规 FF 设计. 它基于一个正规 部分的字长型, 因而主要是组合形式的. 构造 MA 正规 FF 设计的文献很多, 有关 对称因析设计的可参见文献 [2 4], 有关混合水平因析设计的可参见文献 [5 9], 有关分区组因析设计的可参见文献 [10 13] 等.

收稿日期: 2004-11-30; 接受日期: 2005-10-19

* 国家自然科学基金资助项目 (批准号: 10231030, 10571093)

**E-mail: myai@math.pku.edu.cn 
当一些因子的水平很难改变或改变起来费用昂贵时, 对所有试验水平组合 实施完全随机化是不现实的, 而包含两步随机化的 FFSP 设计将是一个可行的设 计方案. 随后的进一步讨论将会表明，一个 FFSP 设计的设计矩阵与一个传统的 FF 设计的设计矩阵没有什么不同，区别在于它有如下两个特殊的性质: (a) 并非 所有的因子有相同的状态，(b) 推断可能在两个不同的准确度水平上进行. 详细 的讨论可参见文献 [14 18]. 由于 FFSP 设计的上述特殊性, 通常会存在多个非同 构的 FFSP 设计同时有 MA. 于是，文献 [19,20] 提出了一个最小附加混杂 (MSA) 的准则, 它显著地缩小了可选的非同构 MA 设计的集合, 而且往往会导出唯一 的最优设计.

我们知道, 用参照设计刻画部分因析设计的特征对于构造近似饱和的因析 设计是十分有效的, 因为此时的参照设计很小. Mukerjee 和方开泰 ${ }^{[20]}$ 在 MA 和 MSA 两个准则下利用补子集来寻找最优的正规对称部分因析裂区 (FFSP) 设计, 不过他们的附加字长型并未用补子集的统一形式表示. 本文试图建立通过参照设 计来识别 MSA 对称或混合水平 FFSP 设计的一般而统一的规则. 对于对称 FFSP 设计而言, 我们的规则等价于文献 [20] 中的结果, 不过我们的表达更加一般, 是 他们结果的进一步改进. 对于混合水平 FFSP 设计而言, 我们的结果是全新的.

\section{2 正规 FFSP $s^{n_{1}-p_{1}} \cdot s^{n_{2}}$ 设计}

\section{1 预备知识和投影几何描述}

考虑 $s^{t}(t=n-p)$ 个水平组合的正规 $\mathrm{FF} s^{n-p}$ 设计, 其中包含 $n$ 个因子 $Z_{1}, \cdots, Z_{n}$, 每个因子有 $s$ 个水平，这里 $s(s \geqslant 2)$ 是一个素数或素数幂. 假设在 这 $n$ 个因子中有 $n_{1}\left(1 \leqslant n_{1}<n\right)$ 个因子的水平改变起来比较困难或者昂贵, 不 妨假设为 $Z_{1}, \cdots, Z_{n_{1}}$, 剩下的 $n_{2}\left(=n-n_{1}\right)$ 个因子是比较容易改变的. 为了节约 可用的资源，假设试验允许安排因子 $Z_{1}, \cdots, Z_{n_{1}}$ 的 $s^{n_{1}-p_{1}}$ 个不同的水平组合， 其中 $0 \leqslant p_{1}<n_{1}$. 令

$$
t_{1}=n_{1}-p_{1}, \quad n_{2}=n-n_{1}, \quad p_{2}=p-p_{1}, \quad t_{2}=t-t_{1} .
$$

由于因子 $Z_{1}, \cdots, Z_{n_{1}}$ 的特殊性质, 在试验的过程中, 很自然地会使用分两个 阶段随机化的裂区结构来代替完全随机化过程. 这一想法可以表述如下: 在因子 $Z_{1}, \cdots, Z_{n_{1}}$ 的 $s^{t_{1}}$ 个不同的水平组合中随机选取一个, 安排因子 $Z_{n_{1}+1}, \cdots, Z_{n}$ 的 $s^{t_{2}}$ 个不同的水平组合，同时保持因子 $Z_{1}, \cdots, Z_{n_{1}}$ 水平不变. 对因子 $Z_{1}, \cdots, Z_{n_{1}}$ 的其他水平组合重复这一过程. 注意到, 安排在因子 $Z_{1}, \cdots, Z_{n_{1}}$ 的不同水平组合 下的因子 $Z_{n_{1}+1}, \cdots, Z_{n}$ 的 $s^{t_{2}}$ 个不同的水平组合不一定要相同. 采用这种方法, 定义了一个 FFSP $s^{\left(n_{1}+n_{2}\right)-\left(p_{1}+p_{2}\right)}$ 设计. 为了与传统的部分因析设计相区别, 上 面描述的 FFSP 设计记为 $s^{n_{1}-p_{1}} \cdot s^{n_{2}}$. 因子 $Z_{1}, \cdots, Z_{n_{1}}$ 的 $s^{t_{1}}$ 个水平组合中的每一 个都定义为一个全区 (WP), 包含的由因子 $Z_{n_{1}+1}, \cdots, Z_{n}$ 变化生成的 $s^{t_{2}}$ 个不同的 水平组合称为子区 $(\mathrm{SP})$. 所以, 因子 $Z_{1}, \cdots, Z_{n_{1}}$ 称为 $\mathrm{WP}$ 因子, 因子 $Z_{n_{1}+1}, \cdots, Z_{n}$ 
称为 SP 因子. 自此以后, 如无特别的说明, 本文中也只考虑那些任意两个主效 应都不别名, 而且任意一个 SP 因子的主效应都不与只包含 WP 因子的交互效应 别名的正规 FFSP 设计.

下面讨论如何用投影几何描述上述 FFSP 设计. 令 $P$ 为 Galois 域 $G F(s)$ 上 的 $(t-1)$ 维有限投影几何 $P G(t-1, s)$ 中的点的集合. $P$ 中的点是 $G F(s)$ 上 $t$ 维 的非零向量. 对于 $P$ 中的任意两点 $x$ 和 $y$, 如果存在某个 $\lambda \in G F(s)$, 使得 $x=\lambda y$, 则称 $x$ 和 $y$ 是相同的. 对于 $P$ 的任意非空子集 $Q$, 令 $V(Q)$ 表示以 $Q$ 中的点的坐 标作为列的矩阵. 对于任意的非负整数 $u$, 记

$$
L_{u}=\left(s^{u}-1\right) /(s-1),
$$

则 $\# P=L_{t}$, 其中 \# 表示集合中元素的个数.

记 $P_{1}$ 为 $P$ 中的一个 $\left(t_{1}-1\right)$ 平面, 且 $P_{2}=P \backslash P_{1}$, 则一个正规的 FFSP $s^{n_{1}-p_{1}}$ $\cdot s^{n_{2}}$ 设计可以由 $P$ 中的一对有序子集 $\left(C_{1}, C_{2}\right)$ 确定, 且这一对有序子集满足:

(a) $\# C_{i}=n_{i}(i=1,2)$,

(b) $C_{i} \subset P_{i}(i=1,2)$,

(c) $\operatorname{rank}\left\{V\left(C_{1}\right)\right\}=t_{1}$,

(d) $\operatorname{rank}\{V(C)\}=t$, 其中 $C=C_{1} \cup C_{2}$.

$P$ 中这样的一对有序子集称作一个合格的 $\left(n_{1}, n_{2}\right)$ 对. 设计的处理水平组合可以 由 $\mathcal{M}[V(C)]$ 中的向量确定, 其中 $\mathcal{M}(\cdot)$ 表示一个矩阵的行空间. 与一个合格的子 集对 $\left(C_{1}, C_{2}\right)$ 相对应的正规 FFSP 设计记为 $d\left(C_{1}, C_{2}\right)$. 考虑到 $C_{1}, C_{2}$ 和 $P_{1}, P_{2}$ 中 的元素个数, 这种构造可行当且仅当

$$
t_{1} \leqslant n_{1}<L_{t_{1}}, \quad t_{2} \leqslant n_{2}<L_{t}-L_{t_{1}},
$$

本节总假定该条件成立.

为了解释一个 FFSP 设计的字长型，我们现在引入 “束” 的概念. 对于一个 有 $s^{t}$ 个水平组合的正规 FFSP $s^{n_{1}-p_{1}} \cdot s^{n_{2}}$ 设计, 一个束就是 $G F(s)$ 上的一个非 零的 $n$ 维向量. 一个有 $i$ 个非零元素的束对应于一个 $i$ 因子交互效应. 一个束 $\xi$ 出现在设计的定义对照子群中, 当且仅当 $V(C) \xi=0$. 不出现在设计的定义对照 子群中的两个不同的束 $\xi^{(i)}(i=1,2)$ 相互别名, 当且仅当 $V(C) \xi^{(1)}$ 和 $V(C) \xi^{(2)}$ 与 $P$ 中的同一个点成比例.

对于 $i=1, \cdots, n$, 令 $A_{i}(d)$ 表示出现在一个正规的 FFSP $s^{n_{1}-p_{1}} \cdot s^{n_{2}}$ 设计 $d\left(C_{1}, C_{2}\right)$ 的定义对照子群中的不同的 $i$ 因子交互效应束的个数. 显然,

$$
A_{1}(d)=A_{2}(d)=0 .
$$

使得 $A_{i}(d)>0$ 的最小整数 $i$ 称作设计 $d$ 的分辨度. 序列

$$
W_{1}(d)=\left(A_{3}(d), \cdots, A_{n}(d)\right)
$$

称作设计 $d$ 的字长型. 对于任意两个正规的 FFSP $s^{n_{1}-p_{1}} \cdot s^{n_{2}}$ 设计 $d_{1}$ 和 $d_{2}$, 令 $u$ 是使得 $A_{i}\left(d_{1}\right) \neq A_{i}\left(d_{2}\right)$ 的最小的整数 $i$. 若 $A_{u}\left(d_{1}\right)<A_{u}\left(d_{2}\right)$, 则称 $d_{1}$ 比 $d_{2}$ 有更 
小的低阶混杂. 若没有其他设计比设计 $d$ 有更小的低阶混杂, 则称设计 $d$ 有最小 低阶混杂 (MA).

由于 WP 因子与 SP 因子之间缺少可交换性, 对于给定的参数 $n_{1}, n_{2}, p_{1}$ 和 $t$, 经常同时存在多个非同构的正规 FFSP $s^{n_{1}-p_{1}} \cdot s^{n_{2}}$ 设计都有 MA. 认识到在一 个 FFSP 中, WP 和 SP 水平的误差有显著的不同, 文献 [20] 提出了一个准则来 区分这些非同构的 MA 设计. 对于一个给定的正规 FFSP $s^{n_{1}-p_{1}} \cdot s^{n_{2}}$ 设计 $d$, 所 有的束被划分为两种类型: 只包含 WP 因子的束称作 WP 型束, 而那些至少包 含一个 SP 因子的束称为 SP 型束. 对 $i=1, \cdots, n$, 令 $B_{i}(d)$ 表示出现在 WP 型 效应别名集中的不同的 SP 型 $i$ 因子交互效应束的个数. 显然有 $B_{1}(d)=0$. 序 列 $W_{2}(d)=\left(B_{2}(d), \cdots, B_{n}(d)\right)$ 称作 $d$ 的附加字长型. 对任意两个非同构的 MA FFSP $s^{n_{1}-p_{1}} \cdot s^{n_{2}}$ 设计 $d_{1}$ 和 $d_{2}$, 令 $u$ 是使得 $B_{i}\left(d_{1}\right) \neq B_{i}\left(d_{2}\right)$ 的最小的整数 $i$. 若 $B_{u}\left(d_{1}\right)<B_{u}\left(d_{2}\right)$, 则称 $d_{1}$ 比 $d_{2}$ 有更小的附加混杂. 一个 MA 设计 $d$ 被称为有最 小附加混杂 (MSA), 如果没有其他的 MA 设计比 $d$ 有更小的附加混杂.

\section{2 与参照设计的联系}

先介绍一些编码理论的概念, 以便后面用于建立 FFSP 设计理论与编码理论 之间的联系. 关于一般关系的细节可以参见文献 [21,22]. 令 $H_{1}$ 表示 $P_{1}$ 中 $t_{1}$ 个 线性独立的点的集合. 对于任意一个非空子集 $Q \subset P$, 令 $d(Q)$ 表示一个设计矩 阵, 其列由与 $Q$ 在 $P$ 中的位置相对应的 $d(P)$ 中的列确定. 既然一个正规设计对 应于编码理论中的一个线性码, 可以用同样的概念表示它们. 对于一个有 $s^{t}$ 个 水平组合的正规 FFSP $s^{n_{1}-p_{1}} \cdot s^{n_{2}}$ 设计 $d\left(C_{1}, C_{2}\right)$, 记 $C=C_{1} \cup C_{2}, d\left(H_{1}, C\right)$ 中的 一个行向量 $a$ 可以写成 $a=\left(a_{1}, a_{2}\right)$, 其中 $a_{1} \in d\left(H_{1}\right), a_{2} \in d(C)$. 令 $E_{i, j}\left[d\left(H_{1}, C\right)\right]$ 表示满足 $w t\left(a_{1}\right)=i, w t\left(a_{2}\right)=j$ 的向量 $a \in d\left(H_{1}, C\right)$ 的个数, 其中 $w t(a)$ 是向 量 $a$ 的 Hamming 重量. 码 $d\left(H_{1}, C\right)$ 和其对偶码 $d\left(H_{1}, C\right)^{\perp}$ 的二元重量分布之间 有如下的关系: 对一切 $j_{1}=0, \cdots, t_{1}, j_{2}=0, \cdots, n$, 有

$$
E_{j_{1}, j_{2}}\left[d\left(H_{1}, C\right)^{\perp}\right]=s^{-t} \sum_{i_{1}=0}^{t_{1}} \sum_{i_{2}=0}^{n} P_{j_{1}}\left(i_{1} ; t_{1}\right) P_{j_{2}}\left(i_{2} ; n\right) E_{i_{1}, i_{2}}\left[d\left(H_{1}, C\right)\right],
$$

其中

$$
P_{j}(k ; n)=\sum_{i=0}^{j}(-1)^{i}(s-1)^{j-i}\left(\begin{array}{c}
k \\
i
\end{array}\right)\left(\begin{array}{c}
n-k \\
j-i
\end{array}\right)
$$

是 Krawtchouk 多项式. 由 Krawtchouk 多项式的正交性, 即

$$
\sum_{j=0}^{n} P_{i}(j ; n) P_{j}(k ; n)=s^{n} \delta_{i, k},
$$

显然有

$$
\begin{array}{r}
E_{j_{1}, j_{2}}\left[d\left(H_{1}, C\right)\right]=s^{t-t_{1}-n} \sum_{i_{1}=0}^{t_{1}} \sum_{i_{2}=0}^{n} P_{j_{1}}\left(i_{1} ; t_{1}\right) P_{j_{2}}\left(i_{2} ; n\right) E_{i_{1}, i_{2}}\left[d\left(H_{1}, C\right)^{\perp}\right], \\
j_{1}=0, \cdots, t_{1}, \quad j_{2}=0, \cdots, n .
\end{array}
$$


(1) 和 (2) 式是所谓的广义 MacWilliams 等式.

对于一个给定的有 $s^{t}$ 个水平组合的正规的 FFSP $s^{n_{1}-p_{1}} \cdot s^{n_{2}}$ 设计 $d=d\left(C_{1}\right.$, $\left.C_{2}\right)$, 令

$$
F_{1}=P_{1} \backslash C_{1}, \quad F_{2}=P_{2} \backslash C_{2},
$$

设计 $d\left(F_{1}, F_{2}\right)$ 对应于一个正规的 FFSP $s^{f_{1}} \cdot s^{f_{2}}$ 设计, 记为 $d_{R}=d\left(F_{1}, F_{2}\right)$, 称作 $d$ 的参照设计, 其中

$$
f_{1}=L_{t_{1}}-n_{1}, \quad f_{2}=L_{t}-L_{t_{1}}-n_{2} .
$$

令 $f=f_{1}+f_{2}$. 文献 [4] 研究了 $W_{1}(d)$ 和 $W_{1}\left(d_{R}\right)$ 之间的关系. 下面推导 $W_{2}(d)$ 和 $W_{2}\left(d_{R}\right)$ 之间的关系.

由于 $d(P)$ 是一个饱和设计, 则它是 Hamming 码的对偶码, 有 $s^{t}-1$ 个向量 的重量是 $s^{t-1}$, 一个向量的重量是零 (文献 [23], p.75), 即 $E_{0}[d(P)]=1$ 且

$$
E_{s^{t-1}}[d(P)]=s^{t}-1 .
$$

由于 $F=F_{1} \cup F_{2}$ 是 $C$ 在 $P$ 中的补子集, 由此可以证明下面的引理 1, 这一引理 给出了 $d\left(H_{1}, C\right)$ 的重量分布和 $d\left(H_{1}, F\right)$ 的重量分布之间的关系:

引理 1 重量分布 $\left\{E_{i_{1}, i_{2}}\left[d\left(H_{1}, C\right)\right]\right\}$ 和 $\left\{E_{i_{1}, i_{2}}\left[d\left(H_{1}, F\right)\right]\right\}$ 满足如下条件:

(i) $E_{i_{1}, i_{2}}\left[d\left(H_{1}, C\right)\right]=0$, 当 $i_{2}>\min \left(s^{t-1}, n\right)$,

(ii) $E_{i_{1}, i_{2}}\left[d\left(H_{1}, F\right)\right]=0$, 当 $i_{2}>\min \left(s^{t-1}, f\right)$,

(iii) $E_{i_{1}, i_{2}}\left[d\left(H_{1}, C\right)\right]=E_{i_{1}, s^{t-1}-i_{2}}\left[d\left(H_{1}, F\right)\right]+I_{\left[i_{2}=0\right]}-I_{\left[i_{2}=s^{t-1}\right]}$, 其他情形,

其中 $I_{[\cdot]}$ 是示性函数, 根据条件 [.] 成立与否取值 1 或者 0 .

结合引理 1 和广义 MacWilliams 等式, 可以得到 $\left\{E_{j_{1}, j_{2}}\left[d\left(H_{1}, C\right)^{\perp}\right]\right\}$ 和 $\left\{E_{j_{1}, j_{2}}\left[d\left(H_{1}, F\right)^{\perp}\right]\right\}$ 之间的如下关系, 这一关系在研究与参照设计之间的关系 时起到重要的作用, 其证明在附录中给出:

引理 2 重量分布 $\left\{E_{j_{1}, j_{2}}\left[d\left(H_{1}, C\right)^{\perp}\right]\right\}$ 和 $\left\{E_{j_{1}, j_{2}}\left[d\left(H_{1}, F\right)^{\perp}\right]\right\}$ 满足如下等 式:

$$
\begin{aligned}
& \sum_{j_{1}=1}^{t_{1}} E_{j_{1}, j_{2}}\left[d\left(H_{1}, C\right)^{\perp}\right] \\
= & \theta_{j_{2}}^{(1)}+\sum_{k_{2}=0}^{f} \theta_{j_{2}, k_{2}}^{(1)}\left\{\sum_{k_{1}=1}^{t_{1}} E_{k_{1}, k_{2}}\left[d\left(H_{1}, F\right)^{\perp}\right]\right\}, \quad j_{2}=0, \cdots, n,
\end{aligned}
$$

其中

$$
\begin{aligned}
& \theta_{j_{2}}^{(1)}=s^{-t}\left(s^{t_{1}}-1\right)\left[P_{j_{2}}(0 ; n)-P_{j_{2}}\left(s^{t-1} ; n\right)\right], \quad j_{2}=0, \cdots, n, \quad k_{2}=0, \cdots, f . \\
& \theta_{j_{2}, k_{2}}^{(1)}=s^{-f} \sum_{i=0}^{f} P_{j_{2}}\left(s^{t-1}-i ; n\right) P_{i}\left(k_{2} ; f\right),
\end{aligned}
$$

特别地,

$$
\theta_{j, j-1}^{(1)}=(-1)^{j}[1+(j-1)(s-2)], \quad \theta_{j, j}^{(1)}=(-1)^{j} .
$$


下面的引理给出了试验设计理论中的字长型与编码理论中重量分布之间的 关系，其证明也在附录中给出：

引理 3 记 $\left\{E_{j, i}\left[d\left(H_{1}, C\right)^{\perp}\right]\right\}$ 和 $\left\{E_{j, i}\left[d\left(H_{1}, F\right)^{\perp}\right]\right\}$ 分别为 $d\left(H_{1}, C\right)^{\perp}$ 和 $d\left(H_{1}\right.$, $F)^{\perp}$ 的重量分布，则

(i)

$$
\sum_{j=1}^{t_{1}} E_{j, i}\left[d\left(H_{1}, C\right)^{\perp}\right]=(s-1)\left[B_{i}(d)-A_{i}\left(C_{1}\right)\right]+\left(\begin{array}{c}
n_{1} \\
i
\end{array}\right)(s-1)^{i},
$$

(ii)

$$
\sum_{j=1}^{t_{1}} E_{j, i}\left[d\left(H_{1}, F\right)^{\perp}\right]=(s-1)\left[B_{i}\left(d_{R}\right)-A_{i}\left(F_{1}\right)\right]+\left(\begin{array}{c}
f_{1} \\
i
\end{array}\right)(s-1)^{i},
$$

其中对于任意的非空子集 $Q \subset P, A_{i}(d(Q))$ 简记为 $A_{i}(Q), B_{i}\left(d_{R}\right)=\#\{\xi: V(F) \xi$ 与 $P_{1}$ 中的某点成比列, $\xi$ 是 SP 型 $i$ 因子交互效应束 $\}$.

由于 $F_{1}$ 是 $C_{1}$ 在 $\left(t_{1}-1\right)$ 平面 $P_{1}$ 中的补子集，由文献 [4] 的定理 3, 得到以 下等式:

$$
A_{i}\left(C_{1}\right)=\theta_{i}^{(2)}+\sum_{l=0}^{i} \theta_{i, l}^{(2)} A_{l}\left(F_{1}\right), \quad i=0, \cdots, n
$$

其中

$$
\begin{aligned}
& \theta_{i}^{(2)}=s^{-t_{1}}\left[P_{i}\left(0 ; n_{1}\right)-P_{i}\left(s^{t_{1}-1} ; n_{1}\right)\right], \\
& \theta_{i, l}^{(2)}=s^{-f_{1}} \sum_{k=0}^{f_{1}} P_{i}\left(s^{t_{1}-1}-k ; n_{1}\right) P_{k}\left(l ; f_{1}\right),
\end{aligned}
$$

由引理 1 3 和 (3) 式得到如下定理, 它建立了一个正规的 FFSP 设计与其参 照设计的附加字长型之间的联系:

定理 1 一个正规的 FFSP $s^{n_{1}-p_{1}} \cdot s^{n_{2}}$ 设计 $d$ 与其参照设计 $d_{R}$ 的附加字长 型 $\left\{B_{i}(d)\right\}$ 和 $\left\{B_{i}\left(d_{R}\right)\right\}$ 之间满足

$$
B_{i}(d)=c_{1}+\sum_{l=0}^{i} \theta_{i, l}^{(1)} B_{l}\left(d_{R}\right)+\sum_{l=0}^{i-2}\left[\theta_{i, l}^{(2)}-\theta_{i, l}^{(1)}\right] A_{l}\left(F_{1}\right), \quad i=2, \cdots, n,
$$

其中常数 $c_{1}$ 只依赖于参数 $t, n, t_{1}, n_{1}$ 和 $s$, 不依赖于 $P$ 中合格的子集对 $\left(C_{1}, C_{2}\right)$ 的选取.

定理 1 是文献 [20] 中定理 3 的改进和推广, 而当 $i=2,3,4$ 时, 所得结论是 等价的.

\section{3 识别 MSA FFSP 设计的规则}

当 $l=0,1,2$ 时, $A_{l}\left(F_{1}\right)=0$, 所以对 $i=2,3,4$, 定理 1 的等式可以简化为如 下的关系:

$$
B_{i}(d)=\text { 常数 }+\sum_{l=2}^{i} \theta_{i, l}^{(1)} B_{l}\left(d_{R}\right) \text {. }
$$


当参照设计的列数较小时, (4) 式可以用来通过参照设计有效地识别 MSA 的 正规对称 FFSP 设计. 注意到, 依次最小化 $d$ 的附加字长型 $\left\{B_{i}(d)\right\}_{2 \leqslant i \leqslant 4}$ 等价于 依次最小化 $d_{R}$ 的 $\left\{(-1)^{i} B_{i}\left(d_{R}\right)\right\}_{2 \leqslant i \leqslant 4}$, 从而得到通过参照设计的字长型和附加 字长型来识别 MSA 正规对称 FFSP 设计的如下规则:

定理 2 令 $\left\{A_{i}(d)\right\},\left\{B_{i}(d)\right\}$ 和 $\left\{A_{i}\left(d_{R}\right)\right\},\left\{B_{i}\left(d_{R}\right)\right\}$ 分别是正规的 FFSP $s^{n_{1}-p_{1}} \cdot s^{n_{2}}$ 设计 $d$ 与其参照设计 $d_{R}$ 的字长型和附加字长型, 则 $d$ 有 MSA 当 且仅当 $d_{R}$ 在同构意义下是依次最小化下面序列的唯一的设计:

$$
\left\{(-1)^{i} A_{i}\left(d_{R}\right)\right\}_{i=3, \cdots, f}, \quad\left\{(-1)^{j} B_{j}\left(d_{R}\right)\right\}_{j=2,3,4} .
$$

\section{3 正规 FFSP $s^{n_{1}-p_{1}} \cdot\left(s^{r}\right) s^{n_{2}}$ 设计}

本节考虑有 $s^{t}$ 个水平组合的正规的 FFSP $s^{n_{1}-p_{1}} \cdot\left(s^{r}\right) s^{n_{2}}$ 设计, 包含 $n_{1}$ 个 $s$ 水平的 WP 因子且有 $s^{n_{1}-p_{1}}$ 个不同的水平组合、一个 $s^{r}$ 水平的 SP 因子 $(r \geqslant 2)$ 和 $n_{2}$ 个 $s$ 水平的 SP 因子, $s^{r}$ 水平的 SP 因子由 $\left(s^{r}-1\right) /(s-1)$ 个 $s$ 水平的 SP 因 子集群生成.

\section{1 预备知识和投影几何描述}

类似于第 2 节, 有 $s^{t}$ 个水平组合的正规 FFSP $s^{n_{1}-p_{1}} \cdot\left(s^{r}\right) s^{n_{2}}$ 设计可以由 $P$ 的一个三元子集组 $\left(C_{1}, C_{0}, C_{2}\right)$ 确定, 满足:
(a) $\# C_{i}=n_{i}(i=1,2)$,
(b) $C_{i} \subset P_{i}(i=1,2)$,
(c) $C_{0}$ 是 $P_{2}$ 的一个 $(r-1)$ 平面,
(d) $C_{2}$ 和 $C_{0}$ 不交,
(e) $\operatorname{rank}\left\{V\left(C_{1}\right)\right\}=t_{1}$,
(f) $\operatorname{rank}\left\{V\left(C_{0} \cup C\right)\right\}=t$, 其中 $C=C_{1} \cup C_{2}$.

$P$ 中这样的有序三元子集组称为一个合格的 $\left(n_{1}, L_{r}, n_{2}\right)$ 组. 设计的处理水平组合 由 $\mathcal{M}\left[V\left(C_{1} \cup C_{0} \cup C_{2}\right)\right]$ 中的向量确定. 与这样一个合格的三元子集组 $\left(C_{1}, C_{0}, C_{2}\right)$ 对应的正规的 FFSP 设计记作 $d\left(C_{1}, C_{0}, C_{2}\right)$. 考虑到 $C_{1}, C_{0}, C_{2}$ 和 $P_{1}, P_{2}$ 中元素 的个数, 这种构造可行当且仅当

$$
t_{1} \leqslant n_{1}<L_{t_{1}}, \quad r \leqslant t-t_{1}, \quad t-t_{1} \leqslant n_{2}+L_{r}<L_{t}-L_{t_{1}},
$$

本节总假定该条件成立.

对于一个有 $s^{t}$ 个水平组合的正规 FFSP $s^{n_{1}-p_{1}} \cdot\left(s^{r}\right) s^{n_{2}}$ 设计, 一个束就是一 个有形式

$$
\xi=\left(\xi_{1}, \cdots, \xi_{n_{1}}, \xi_{n_{1}+1}, \cdots, \xi_{n_{1}+L_{r}}, \xi_{n_{1}+L_{r}+1}, \cdots, \xi_{n_{1}+L_{r}+n_{2}}\right)^{\mathrm{T}}
$$

的非零向量, 其中 $\xi_{i} \in G F(s)\left(i=1, \cdots, n_{1}+L_{r}+n_{2}\right)$, 而且 $\xi_{n_{1}+1}, \cdots, \xi_{n_{1}+L_{r}}$ 中 至多有一个非零. 一个束 $\xi$ 出现在 $d$ 的定义对照子群中当且仅当

$$
V\left(C_{1} \cup C_{0} \cup C_{2}\right) \xi=0 .
$$


不出现在 $d$ 的定义对照子群中的不同的束 $\xi^{(i)}(i=1,2)$ 互相别名, 当且仅当 $V\left(C_{1} \cup C_{0} \cup C_{2}\right) \xi^{(1)}$ 和 $V\left(C_{1} \cup C_{0} \cup C_{2}\right) \xi^{(2)}$ 与 $P$ 中的同一个点成比例.

正如文献 [5] 中所做, 我们把束划分为两个类: 0 类和 1 类. 一个束 $\xi$ 满足

$$
\xi_{n_{1}+1}=\cdots=\xi_{n_{1}+L_{r}}=0
$$

是 0 类的, 否则是 1 类的. 记 $n=n_{1}+n_{2}$. 对 $i=1, \cdots, n+1$ 和 $j=0,1$, 令 $A_{i, j}(d)$ 表示出现在 $d$ 的定义对照子群中的不同的 $j$ 类 $i$ 因子交互效应束的个数.

对 $j=0,1$, 显然有

$$
A_{1, j}(d)=A_{2, j}(d)=0
$$

序列

$$
W_{1}(d)=\left\{\left(A_{i, 0}(d), A_{i, 1}(d)\right)\right\}_{3 \leqslant i \leqslant n+1}
$$

称为设计 $d$ 的字长型. 对任意两个正规的 FFSP $s^{n_{1}-p_{1}} \cdot\left(s^{r}\right) s^{n_{2}}$ 设计 $d_{1}$ 和 $d_{2}$, 令 $u$ 是使得

$$
\left(A_{i, 0}\left(d_{1}\right), A_{i, 1}\left(d_{1}\right)\right) \neq\left(A_{i, 0}\left(d_{2}\right), A_{i, 1}\left(d_{2}\right)\right)
$$

的最小的整数 $i$. 如果 (a) $A_{u, 0}\left(d_{1}\right)<A_{u, 0}\left(d_{2}\right)$, 或者 (b) $A_{u, 0}\left(d_{1}\right)=A_{u, 0}\left(d_{2}\right)$ 且 $A_{u, 1}\left(d_{1}\right)<A_{u, 1}\left(d_{2}\right)$, 则称 $d_{1}$ 比 $d_{2}$ 有较小的低阶混杂. 一个 FFSP 设计 $d$ 有 MA, 如果没有其他 FFSP 设计比 $d$ 有更小的低阶混杂.

对于一个给定的正规 FFSP $s^{n_{1}-p_{1}} \cdot\left(s^{r}\right) s^{n_{2}}$ 设计 $d$, 所有的束可以进一步划分 为两种类型. 只包含 WP 因子的束称为 WP 型束, 而至少包含一个 SP 因子的称 为 SP 型束. 对 $i=1, \cdots, n+1$ 和 $j=0,1$, 令 $B_{i, j}(d)$ 表示出现在 WP 型效应别名 集中的不同的 $j$ 类 $\mathrm{SP}$ 型 $i$ 因子交互效应束的个数. 显然有

$$
B_{1,0}(d)=B_{1,1}(d)=0 .
$$

序列

$$
W_{2}(d)=\left\{\left(B_{i, 0}(d), B_{i, 1}(d)\right)\right\}_{2 \leqslant i \leqslant n+1}
$$

称作 $d$ 的附加字长型. 对任意两个非同构的 MA FFSP $s^{n_{1}-p_{1}} \cdot\left(s^{r}\right) s^{n_{2}}$ 设计 $d_{1}$ 和 $d_{2}$, 令 $u$ 是使得

$$
\left(B_{i, 0}\left(d_{1}\right), B_{i, 1}\left(d_{1}\right)\right) \neq\left(B_{i, 0}\left(d_{2}\right), B_{i, 1}\left(d_{2}\right)\right)
$$

的最小的整数 $i$. 如果 (a) $B_{u, 0}\left(d_{1}\right)<B_{u, 0}\left(d_{2}\right)$, 或者 (b) $B_{u, 0}\left(d_{1}\right)=B_{u, 0}\left(d_{2}\right)$ 且 $B_{u, 1}\left(d_{1}\right)<B_{u, 1}\left(d_{2}\right)$, 则称 $d_{1}$ 比 $d_{2}$ 有更小的附加混杂. 一个 MA FFSP 设计 $d$ 有 MSA, 如果没有其他的 MA FFSP 设计比 $d$ 有更小的附加混杂.

\section{2 与参照设计的联系}

对一个有 $s^{t}$ 个水平组合的正规 FFSP $s^{n_{1}-p_{1}} \cdot\left(s^{r}\right) s^{n_{2}}$ 设计 $d=d\left(C_{1}, C_{0}, C_{2}\right)$, 令 $C=C_{1} \cup C_{2} \cdot d\left(H_{1}, C_{0} \cup C\right)$ 中的行向量 $a$ 可以写作 $a=\left(a_{1}, a_{2}, a_{3}\right)$, 其中 $a_{1} \in d\left(H_{1}\right), a_{2} \in d\left(C_{0}\right)$ 且 $a_{3} \in d(C)$, 令 $E_{j, i_{1}, i_{2}}\left[d\left(H_{1}, C_{0} \cup C\right)\right]$ 表示满足 $w t\left(a_{1}\right)=j$ 
和 $w t\left(a_{k}\right)=i_{k}(k=1,2)$ 的向量 $a \in d\left(H_{1}, C_{0} \cup C\right)$ 的个数. $d\left(H_{1}, C_{0} \cup C\right)$ 与其对 偶码 $d\left(H_{1}, C_{0} \cup C\right)^{\perp}$ 的三元重量分布之间的关系可以表示为

$$
\begin{aligned}
& E_{k, j_{1}, j_{2}}\left[d\left(H_{1}, C_{0} \cup C\right)^{\perp}\right] \\
= & s^{-t} \sum_{j=0}^{t_{1}} \sum_{i_{1}=0}^{L_{r}} \sum_{i_{2}=0}^{n} P_{k}\left(j ; t_{1}\right) P_{j_{1}}\left(i_{1} ; L_{r}\right) P_{j_{2}}\left(i_{2} ; n\right) E_{j, i_{1}, i_{2}}\left[d\left(H_{1}, C_{0} \cup C\right)\right], \\
& k=0, \cdots, t_{1}, \quad j_{1}=0, \cdots, L_{r}, \quad j_{2}=0, \cdots, n .
\end{aligned}
$$

由 Krawtchouk 多项式的正交性, 容易推导出 (6) 式的逆向公式, 它们也称作广义 MacWilliams 等式.

对于上述 FFSP 设计 $d=d\left(C_{1}, C_{0}, C_{2}\right)$, 令

$$
F_{1}=P_{1} \backslash C_{1}, \quad F_{2}=P_{2} \backslash\left(C_{0} \cup C_{2}\right),
$$

设计 $d\left(F_{1}, C_{0}, F_{2}\right)$ 对应于一个正规的 FFSP $s^{f_{1}} \cdot\left(s^{r}\right) s^{f_{2}}$ 设计, 记为

$$
d_{R}=d\left(F_{1}, C_{0}, F_{2}\right),
$$

称作 $d$ 的参照设计, 其中

$$
f_{1}=L_{t_{1}}-n_{1}, \quad f_{2}=L_{t}-L_{t_{1}}-L_{r}-n_{2} .
$$

令

$$
F=F_{1} \cup F_{2}, \quad f=f_{1}+f_{2} .
$$

文献 [9] 研究了 $W_{1}(d)$ 和 $W_{1}\left(d_{R}\right)$ 之间的关系, 下面推导 $W_{2}(d)$ 和 $W_{2}\left(d_{R}\right)$ 之间 的关系.

类似于第 2 节中的讨论, 我们得到以下几个引理:

引理 4 三元重量分布 $\left\{E_{j, i_{1}, i_{2}}\left[d\left(H_{1}, C_{0} \cup C\right)\right]\right\}$ 和 $\left\{E_{j, i_{1}, i_{2}}\left[d\left(H_{1}, C_{0} \cup F\right)\right]\right\}$ 满足如下条件:

(i) $E_{j, i_{1}, i_{2}}\left[d\left(H_{1}, C_{0} \cup C\right)\right]=0$, 当 $i_{1}>\min \left(s^{t-1}, L_{r}\right)$ 或 $i_{2}>\min \left(s^{t-1}, n\right)$,

(ii) $E_{j, i_{1}, i_{2}}\left[d\left(H_{1}, C_{0} \cup F\right)\right]=0$, 当 $i_{1}>\min \left(s^{t-1}, L_{r}\right)$ 或 $i_{2}>\min \left(s^{t-1}, f\right)$,

(iii)

$$
\begin{aligned}
& E_{j, i_{1}, i_{2}}\left[d\left(H_{1}, C_{0} \cup C\right)\right] \\
= & B_{j, i_{1}, s^{t-1}-i_{1}-i_{2}}\left[d\left(H_{1}, C_{0} \cup F\right)\right]+I_{\left[i_{1}=i_{2}=0\right]}-I_{\left[i_{1}=0, i_{2}=s^{t-1}\right]}, \quad \text { 其他. }
\end{aligned}
$$

引理 5 三元重量分布 $\left\{E_{k, j_{1}, j_{2}}\left[d\left(H_{1}, C_{0} \cup C\right)^{\perp}\right]\right\}$ 和 $\left\{E_{k, j_{1}, j_{2}}\left[d\left(H_{1}, C_{0} \cup\right.\right.\right.$ $\left.\left.F)^{\perp}\right]\right\}$ 满足

$$
\begin{aligned}
& \sum_{k=1}^{t_{1}} \sum_{j_{1}=0}^{L_{r}} E_{k, j_{1}, j_{2}}\left[d\left(H_{1}, C_{0} \cup C\right)^{\perp}\right] \\
= & s^{L_{r}} \theta_{j_{2}}^{(1)}+\sum_{i_{2}=0}^{f} \theta_{j_{2}, i_{2}}^{(1)}\left\{\sum_{j=1}^{t_{1}} \sum_{i_{1}=0}^{L_{r}} E_{j, i_{1}, i_{2}}\left[d\left(H_{1}, C_{0} \cup F\right)^{\perp}\right]\right\}, \quad j_{2}=0, \cdots, n .
\end{aligned}
$$

引理 6 记 $\left\{E_{j, i_{1}, i_{2}}\left[d\left(H_{1}, C_{0} \cup C\right)^{\perp}\right]\right\}$ 和 $\left\{E_{j, i_{1}, i_{2}}\left[d\left(H_{1}, C_{0} \cup F\right)^{\perp}\right]\right\}$ 分别是 $d\left(H_{1}, C_{0} \cup C\right)^{\perp}$ 和 $d\left(H_{1}, C_{0} \cup F\right)^{\perp}$ 的三元重量分布, 则 
(i)

$\sum_{j=1}^{t_{1}} E_{j, 0, i_{2}}\left[d\left(H_{1}, C_{0} \cup C\right)^{\perp}\right]=(s-1)\left[B_{i_{2}, 0}(d)-A_{i_{2}}\left(C_{1}\right)\right]+\left(\begin{array}{c}n_{1} \\ i_{2}\end{array}\right)(s-1)^{i_{2}}$,

(ii)

$$
\sum_{j=1}^{t_{1}} E_{j, 1, i_{2}}\left[d\left(H_{1}, C_{0} \cup C\right)^{\perp}\right]=(s-1) B_{i_{2}+1,1}(d),
$$

(iii)

$$
\sum_{j=1}^{t_{1}} E_{j, 0, i_{2}}\left[d\left(H_{1}, C_{0} \cup F\right)^{\perp}\right]=(s-1)\left[B_{i_{2}, 0}\left(d_{R}\right)-A_{i_{2}}\left(F_{1}\right)\right]+\left(\begin{array}{c}
f_{1} \\
i_{2}
\end{array}\right)(s-1)^{i_{2}},
$$

(iv)

$$
\sum_{j=1}^{t_{1}} E_{j, 1, i_{2}}^{\prime}\left[d\left(H_{1}, C_{0} \cup F\right)^{\perp}\right]=(s-1) B_{i_{2}+1,1}\left(d_{R}\right),
$$

其中 $B_{i, u}\left(d_{R}\right)=\#\left\{\xi: V\left(C_{0} \cup F\right) \xi\right.$ 与 $P_{1}$ 中的某点成比例, $\xi$ 是一个 $u$ 类 SP 型 $i$ 因子交互效应束 $\}$.

对一切 $i=0, \cdots, L_{r}$, 令 $\gamma_{r}(i)=E_{i}\left[d\left(C_{0}\right)^{\perp}\right)$. 由 MacWilliams 等式可知

$$
\gamma_{r}(i)=s^{-r} \sum_{j=0}^{L_{r}} P_{i}\left(j ; L_{r}\right) E_{j}\left[d\left(C_{0}\right)\right]=s^{-r}\left[P_{i}\left(0 ; L_{r}\right)+P_{i}\left(s^{r-1} ; L_{R}\right)\left(s^{r}-1\right)\right] \text {. }
$$

令 $V_{i}$ 表示 $i$ 维线性向量空间, $d\left(C_{0}\right)$ 在 $V_{1} \times V_{L_{r}-1}$ 中分块的二元重量分布 $\left\{E_{i_{1}, i_{2}}\left[d\left(C_{0}\right)\right]\right\}$ 是

$$
\begin{gathered}
E_{0,0}\left[d\left(C_{0}\right)\right]=1, \quad E_{0, s^{r-1}}\left[d\left(C_{0}\right)\right]=s^{r-1}-1, \\
E_{1, s^{r-1}-1}\left[d\left(C_{0}\right)\right]=s^{r-1}(s-1),
\end{gathered}
$$

且对其他的 $i_{1}$ 和 $i_{2}$, 有 $E_{i_{1}, i_{2}}\left[d\left(C_{0}\right)\right]=0$.

令

$$
\alpha_{r}(i)= \begin{cases}E_{1, i}\left[d\left(C_{0}\right)^{\perp}\right], & 0 \leqslant i \leqslant L_{r}, i \neq 1, \\ 1, & i=1 .\end{cases}
$$

沿用文献 [10] 中引理 2 的推理, 可以得到

引理 7 记 $\left\{E_{j, i_{1}, i_{2}}\left[d\left(H_{1}, C_{0} \cup C\right)^{\perp}\right]\right\}$ 和 $\left\{E_{j, i_{1}, i_{2}}\left[d\left(H_{1}, C_{0} \cup F\right)^{\perp}\right]\right\}$ 分别是 $d\left(H_{1}, C_{0} \cup C\right)^{\perp}$ 和 $d\left(H_{1}, C_{0} \cup F\right)^{\perp}$ 的三元重量分布, 则对一切 $j=0, \cdots, t_{1}, i_{1}=$ $0, \cdots, L_{r}$, 有

(i)

$$
\begin{aligned}
& E_{j, i_{1}, i_{2}}\left[d\left(H_{1}, C_{0} \cup C\right)^{\perp}\right] \\
= & \alpha_{r}\left(i_{1}\right) E_{j, 1, i_{2}}\left[d\left(H_{1}, C_{0} \cup C\right)^{\perp}\right]+\gamma_{r}\left(i_{1}\right) E_{j, 0, i_{2}}\left[d\left(H_{1}, C_{0} \cup C\right)^{\perp}\right],
\end{aligned}
$$

(ii)

$$
\begin{aligned}
& E_{j, i_{1}, i_{2}}\left[d\left(H_{1}, C_{0} \cup F\right)^{\perp}\right] \\
= & \alpha_{r}\left(i_{1}\right) E_{j, 1, i_{2}}\left[d\left(H_{1}, C_{0} \cup F\right)^{\perp}\right]+\gamma_{r}\left(i_{1}\right) E_{j, 0, i_{2}}\left[d\left(H_{1}, C_{0} \cup F\right)^{\perp}\right] .
\end{aligned}
$$


显然,

$$
\sum_{i=0}^{L_{r}} \alpha_{r}(i)=(s-1) s^{L_{r}-r-1}+1, \quad \sum_{i=0}^{L_{r}} \gamma_{r}(i)=s^{L_{r}-r} .
$$

总结上述引理可得到

定理 3 一个正规的 FFSP $s^{n_{1}-p_{1}} \cdot\left(s^{r}\right) s^{n_{2}}$ 设计 $d=d\left(C_{1}, C_{0}, C_{2}\right)$ 与其参照 设计 $d_{R}$ 的附加字长型 $\left\{B_{i, j}(d)\right\}$ 和 $\left\{B_{i, j}\left(d_{R}\right)\right\}$ 满足

$$
\begin{aligned}
B_{i, 0}(d)+\eta B_{i+1,1}(d)= & c_{2}+\sum_{j=0}^{i} \theta_{i, j}^{(1)}\left[B_{j, 0}\left(d_{R}\right)+\eta B_{j+1,1}\left(d_{R}\right)\right] \\
& +\sum_{j=0}^{i-2}\left(\theta_{i, j}^{(2)}-\theta_{i, j}^{(1)}\right) A_{j}\left(F_{1}\right), \quad i=1, \cdots, n+1,
\end{aligned}
$$

其中

$$
\eta=s^{r-L_{r}}\left[(s-1) s^{L_{r}-r-1}+1\right],
$$

常数 $c_{2}$ 只与参数 $t, n, t_{1}, n_{1}, r$ 和 $s$ 有关, 与 $P$ 中的三元合格子集组 $\left(C_{1}, C_{0}, C_{2}\right)$ 的选取无关.

对有 $s^{t}$ 个水平组合的正规的 FFSP $s^{n_{1}-p_{1}} \cdot\left(s^{r}\right) s^{n_{2}}$ 设计 $d=d\left(C_{1}, C_{0}, C_{2}\right)$, $B_{i, 0}(d)=B_{i}\left[d\left(C_{1}, C_{2}\right)\right]$ 是正规对称 FFSP $s^{n_{1}-p_{1}} \cdot s^{n_{2}}$ 设计 $d\left(C_{1}, C_{2}\right)$ 的附加字长 型. 由定理 1 , 它们可以用设计 $d\left(C_{1}, C_{2}\right)$ 的参照设计 $d\left(F_{1}, C_{0} \cup F_{2}\right)$ 的附加字长型 表示出来. 另一方面, $d\left(F_{1}, C_{0} \cup F_{2}\right)$ 可以看作 $d$ 的参照设计 $d_{R}$. 于是我们可以得 到如下的定理, 其证明在附录中给出:

定理 4 记 $\left\{B_{i, j}(d)\right\}$ 和 $\left\{B_{i, j}\left(d_{R}\right)\right\}$ 分别是一个正规的 FFSP $s^{n_{1}-p_{1}} \cdot\left(s^{r}\right) s^{n_{2}}$ 设计 $d=d\left(C_{1}, C_{0}, C_{2}\right)$ 与其参照设计 $d_{R}$ 的附加字长型, 则有

$$
\begin{aligned}
& B_{i, 0}(d) \\
= & c_{3}+\sum_{k=0}^{i}\left[\sum_{j=k}^{i} \theta_{i, j}^{(3)} \alpha_{r}(j-k)\right] B_{k+1,1}\left(d_{R}\right)+\sum_{k=0}^{i}\left[\sum_{j=k}^{i} \theta_{i, j}^{(3)} \gamma_{r}(j-k)\right] B_{k, 0}\left(d_{R}\right) \\
& +\sum_{k=0}^{i-2}\left[\theta_{i, k}^{(2)}-\sum_{j=k}^{i} \theta_{i, j}^{(3)} \gamma_{r}(j-k)\right] A_{k}\left(F_{1}\right), \quad i=2, \ldots, n+1,
\end{aligned}
$$

其中

$$
\theta_{i, j}^{(3)}=s^{-L_{r}-f} \sum_{k=0}^{L_{r}+f} P_{i}\left(s^{t-1}-k ; n\right) P_{k}\left(j ; L_{r}+f\right),
$$

常数 $c_{3}$ 只与参数 $t, n, t_{1}, n_{1}, r$ 和 $s$ 有关.

\section{3 识别 MSA FFSP 设计的规则}

注意到

$$
\begin{gathered}
\alpha_{r}(0)=0, \quad \alpha_{r}(1)=1, \quad \alpha_{r}(2)=B_{1,2}\left(H_{r}^{\perp}\right), \\
\gamma_{r}(0)=1, \quad \gamma_{r}(1)=0, \quad \gamma_{r}(2)=0,
\end{gathered}
$$


定理 4 可以简化为如下关系:

$$
B_{i, 0}(d)=\text { 常数 }+(-1)^{i}\left[B_{i, 0}\left(d_{R}\right)+B_{i, 1}\left(d_{R}\right)\right]+\text { 低阶项, } \quad i=2,3,4,
$$

由定理 3 和 (9) 式, 依次最小化 $d$ 的附加字长型 $\left\{\left[B_{i, 0}(d), B_{i, 1}(d)\right]\right\}_{i=2,3,4}$ 等 价于依次最小化 $d_{R}$ 的下面的成分:

$$
\left\{(-1)^{i}\left[B_{i, 0}\left(d_{R}\right)+B_{i, 1}\left(d_{R}\right)\right],(-1)^{i-1} B_{i, 1}\left(d_{R}\right)\right\}_{i=2,3,4},
$$

也等价于依次最小化

$$
\left\{(-1)^{i}\left[B_{i, 0}\left(d_{R}\right)+B_{i, 1}\left(d_{R}\right)\right],(-1)^{i} B_{i, 0}\left(d_{R}\right)\right\}_{i=2,3,4},
$$

因此, 结合文献 [9] 中的定理 3, 可以得到下面的通过参照设计的字长型来识别 MSA 正规 FFSP $s^{n_{1}-p_{1}} \cdot\left(s^{r}\right) s^{n_{2}}$ 设计的一般规则:

定理 5 记 $\left\{A_{i, j}(d)\right\},\left\{B_{i, j}(d)\right\}$ 和 $\left\{A_{i, j}\left(d_{R}\right)\right\},\left\{B_{i, j}\left(d_{R}\right)\right\}$ 分别是正规的 FFSP $s^{n_{1}-p_{1}} \cdot\left(s^{r}\right) s^{n_{2}}$ 设计 $d$ 与其参照设计 $d_{R}$ 的字长型和附加字长型, 则 $d$ 有 MSA, 当且仅当 $d_{R}$ 在同构意义下是依次最小化下列序列的唯一的设计:

$$
\begin{aligned}
& \left\{(-1)^{i}\left[A_{i, 0}\left(d_{R}\right)+A_{i, 1}\left(d_{R}\right)\right],(-1)^{i} A_{i, 0}\left(d_{R}\right)\right\}_{i=3, \cdots, f}, \\
& \left\{(-1)^{j}\left[B_{j, 0}\left(d_{R}\right)+B_{j, 1}\left(d_{R}\right)\right],(-1)^{j} B_{j, 0}\left(d_{R}\right)\right\}_{j=2,3,4} .
\end{aligned}
$$

\section{4 正规 FFSP $s^{n_{1}-p_{1}} \cdot\left(s^{r_{1}}\right)\left(s^{r_{2}}\right) s^{n_{2}}$ 设计}

本节考虑有 $s^{t}$ 个水平组合的正规的 FFSP $s^{n_{1}-p_{1}} \cdot\left(s^{r_{1}}\right)\left(s^{r_{2}}\right) s^{n_{2}}$ 设计，包含 $n_{1}$ 个 $s$ 水平的 WP 因子且有 $s^{n_{1}-p_{1}}$ 个不同的水平组合、一个 $s^{r_{1}}$ 水平的 SP 因子 和一个 $s^{r_{2}}$ 水平的 SP 因子 $\left(r_{1} \geqslant 2, r_{2} \geqslant 2\right)$ 和 $n_{2}$ 个 $s$ 水平的 SP 因子, $s^{r_{1}}$ 水平的 SP 因子和 $s^{r_{2}}$ 水平的 SP 因子分别由互斥的 $\left(s^{r_{1}}-1\right) /(s-1)$ 个不同的 $s$ 水平因子 和 $\left(s^{r_{2}}-1\right) /(s-1)$ 个不同的 $s$ 水平因子集群生成.

\section{1 预备知识和投影几何描述}

一个有 $s^{t}$ 个水平组合的正规 FFSP $s^{n_{1}-p_{1}} \cdot\left(s^{r_{1}}\right)\left(s^{r_{2}}\right) s^{n_{2}}$ 设计可以由 $P$ 中的 四元子集组 $\left(C_{1}, C_{01}, C_{02}, C_{2}\right)$ 确定, 满足:
(a) $\# C_{i}=n_{i}(i=1,2)$,
(b) $C_{i} \subset P_{i}(i=1,2)$,
(c) $C_{01}$ 和 $C_{02}$ 分别是 $P_{2}$ 的 $\left(r_{1}-1\right)$ 平面和 $\left(r_{2}-1\right)$ 平面,
(d) $C_{2}, C_{01}$ 和 $C_{02}$ 是不交的,
(e) $\operatorname{rank}\left\{V\left(C_{1}\right)\right\}=t_{1}$,
(f) $\operatorname{rank}\left\{V\left(C_{01} \cup C_{02} \cup C\right)\right\}=t$, 其中 $C=C_{1} \cup C_{2}$.

$P$ 中这样的一组有序的四元子集组称为一个合格的四元组. 设计的处理水平组合 由 $\mathcal{M}\left[V\left(C_{1} \cup C_{01} \cup C_{02} \cup C_{2}\right)\right]$ 中的向量确定. 对应于这样一个合格的四元子集组 $\left(C_{1}, C_{01}, C_{02}, C_{2}\right)$ 的正规 FFSP 设计记为 $d\left(C_{1}, C_{01}, C_{02}, C_{2}\right)$. 考虑到 $C_{1}, C_{01}, C_{02}$, $C_{2}$ 和 $P_{1}, P_{2}$ 的元素个数, 这种构造可行当且仅当

$$
t_{1} \leqslant n_{1}<L_{t_{1}}, \quad \max \left\{r_{1}, r_{2}\right\} \leqslant t-t_{1}, \quad t-t_{1} \leqslant n_{2}+L_{r_{1}}+L_{r-2}<L_{t}-L_{t_{1}},
$$


本节总假定该条件成立. 一个束是一个有形式

$$
\xi=\left(\xi_{1}, \cdots, \xi_{n_{1}+L_{r_{1}}+L_{r_{2}}+n_{2}}\right)^{\mathrm{T}}
$$

的非零向量, 其中 $\xi_{i} \in G F(s)\left(i=1, \cdots, n_{1}+L_{r_{1}}+L_{r_{2}}+n_{2}\right)$, 且在 $\xi_{n_{1}+1}, \cdots, \xi_{n_{1}+L_{r}}$ 中至多一个非零, 在 $\xi_{n_{1}+L_{r_{1}}+1}, \cdots, \xi_{n_{1}+L_{r_{1}}+L_{r_{2}}}$ 中至多一个非零.

不失一般性, 假设 $r_{1} \geqslant r_{2}$. 首先考虑情形 $r_{1}>r_{2}$. 一个束 $\xi$ 出现在 $d$ 的定义 对照子群中, 当且仅当

$$
V\left(C_{1} \cup C_{01} \cup C_{02} \cup C_{2}\right) \xi=0 .
$$

任何这样的束只能包含 $s$ 水平因子, 或者 $s^{r_{2}}$ 水平因子和一些 $s$ 水平因子, 或者 $s^{r_{1}}$ 水平因子和一些 $s$ 水平因子, 或者 $s^{r_{i}}$ 水平因子 $(i=1,2)$ 和一些 $s$ 水平因子.

这 4 种类型的束分别称为 0 类、 1 类、 2 类和 3 类. 令 $n=n_{1}+n_{2}$. 对 $i=3, \cdots, n+2$ 和 $j=0,1,2,3$, 令 $A_{i, j}(d)$ 表示出现在 $d$ 的定义对照子群中的不同的 $j$ 类 $i$ 因子交 互效应束的个数. 序列

$$
W_{1}(d)=\left\{\left(A_{i, 0}(d), A_{i, 1}(d), A_{i, 2}(d), A_{i, 3}(d)\right)\right\}_{3 \leqslant i \leqslant n+2}
$$

称作 $d$ 的字长型. 对任意两个正规 FFSP $s^{n_{1}-p_{1}} \cdot\left(s^{r_{1}}\right)\left(s^{r_{2}}\right) s^{n_{2}}$ 设计 $d_{1}$ 和 $d_{2}$, 令 $u$ 是使得

$$
\left(A_{i, 0}\left(d_{1}\right), A_{i, 1}\left(d_{1}\right), A_{i, 2}\left(d_{1}\right), A_{i, 3}\left(d_{1}\right)\right) \neq\left(A_{i, 0}\left(d_{2}\right), A_{i, 1}\left(d_{2}\right), A_{i, 2}\left(d_{2}\right), A_{i, 3}\left(d_{2}\right)\right)
$$

的最小的整数 $i$. 若下列之一成立, 则称 $d_{1}$ 比 $d_{2}$ 有更小的低阶混杂:

(a) $A_{u, 0}\left(d_{1}\right)<A_{u, 0}\left(d_{2}\right)$,

(b) $A_{u, 0}\left(d_{1}\right)=A_{u, 0}\left(d_{2}\right)$ 且 $A_{u, 1}\left(d_{1}\right)<A_{u, 1}\left(d_{2}\right)$,

(c) $A_{u, 0}\left(d_{1}\right)=A_{u, 0}\left(d_{2}\right), A_{u, 1}\left(d_{1}\right)=A_{u, 1}\left(d_{2}\right)$ 且 $A_{u, 2}\left(d_{1}\right)<A_{u, 2}\left(d_{2}\right)$,

(d) $A_{u, 0}\left(d_{1}\right)=A_{u, 0}\left(d_{2}\right), A_{u, 1}\left(d_{1}\right)=A_{u, 1}\left(d_{2}\right), A_{u, 2}\left(d_{1}\right)=A_{u, 2}\left(d_{2}\right)$ 且 $A_{u, 3}\left(d_{1}\right)$ $<A_{u, 3}\left(d_{2}\right)$.

一个 FFSP 设计 $d$ 有 MA, 如果没有其他 FFSP 设计比 $d$ 有更小的低阶混杂.

对于一个给定的正规 FFSP $s^{n_{1}-p_{1}} \cdot\left(s^{r_{1}}\right)\left(s^{r_{2}}\right) s^{n_{2}}$ 设计 $d$, 正如前文, 所有的 束被进一步划分为两种类型. 只包含 WP 因子的称为 WP 型束, 而包含至少一个 $\mathrm{SP}$ 因子的称为 SP 型束. 对 $i=2, \cdots, n+2$ 和 $j=0,1,2,3$, 令 $B_{i, j}(d)$ 表示出现在 $\mathrm{WP}$ 型效应别名集中的不同的 $j$ 类 SP 型 $i$ 因子交互效应束的个数. 序列

$$
W_{2}(d)=\left\{\left(B_{i, 0}(d), B_{i, 1}(d), B_{i, 2}(d), B_{i, 3}(d)\right)\right\}_{2 \leqslant i \leqslant n+2}
$$

称为 $d$ 的附加字长型. 这样, 我们可以类似定义一个 MA FFSP $s^{n_{1}-p_{1}} \cdot\left(s^{r_{1}}\right)\left(s^{r_{2}}\right) s^{n_{2}}$ 设计进一步有 MSA.

\section{2 与参照设计的联系}

对于一个给定的有 $s^{t}$ 个水平组合的正规 FFSP $s^{n_{1}-p_{1}} \cdot\left(s^{r_{1}}\right)\left(s^{r_{2}}\right) s^{n_{2}}$ 设计 $d=$ $d\left(C_{1}, C_{01}, C_{02}, C_{2}\right)$, 令

$$
F_{1}=P_{1} \backslash C_{1}, \quad F_{2}=P_{2} \backslash\left(C_{01} \cup C_{02} \cup C_{2}\right),
$$


设计 $d\left(F_{1}, C_{01}, C_{02}, F_{2}\right)$ 对应于一个正规 FFSP $s^{f_{1}} \cdot\left(s^{r_{1}}\right)\left(s^{r_{2}}\right) s^{f_{2}}$ 设计, 记为 $d_{R}=$ $d\left(F_{1}, C_{01}, C_{02}, F_{2}\right)$, 称为 $d$ 的参照设计, 其中

$$
f_{1}=L_{t_{1}}-n_{1}, \quad f_{2}=L_{t}-L_{t_{1}}-L_{r_{1}}-L_{r_{2}}-n_{2} .
$$

令

$$
F=F_{1} \cup F_{2}, \quad f=f_{1}+f_{2} .
$$

我们可类似定义这些设计的四元重量分布和对应的 MacWilliams 等式, 在此省略 细节. 文献 [9] 研究了 $W_{1}(d)$ 和 $W_{1}\left(d_{R}\right)$ 之间的关系, 下面仿照第 3 节中的讨论 建立 $W_{2}(d)$ 和 $W_{2}\left(d_{R}\right)$ 之间的关系.

引理 8 四元重量分布 $\left\{E_{k, j_{1}, j_{2}, j_{3}}\left[d\left(H_{1}, C_{01} \cup C_{02} \cup C\right)^{\perp}\right]\right\}$ 和 $\left\{E_{k, j_{1}, j_{2}, j_{3}}\left[d\left(H_{1}\right.\right.\right.$, $\left.\left.\left.C_{01} \cup C_{02} \cup F\right)^{\perp}\right]\right\}$ 满足

$$
\begin{aligned}
& \sum_{k=1}^{t_{1}} \sum_{j_{1}=0}^{L_{r_{1}}} \sum_{j_{2}=0}^{L_{r_{2}}} E_{k, j_{1}, j_{2}, j_{3}}\left[d\left(H_{1}, C_{01} \cup C_{02} \cup C\right)^{\perp}\right] \\
&= s^{L_{r_{1}}+L_{r_{2}}} \theta_{j_{3}}^{(1)}+\sum_{i_{3}=0}^{j_{3}} \theta_{j_{3}, i_{3}}^{(1)}\left\{\sum_{j=1}^{t_{1}} \sum_{i_{1}=0}^{L_{r_{1}}} \sum_{i_{2}=0}^{L_{r_{2}}} E_{j, i_{1}, i_{2}, i_{3}}\left[d\left(H_{1}, C_{01} \cup C_{02} \cup F\right)^{\perp}\right]\right\}, \\
& j_{3}=0, \cdots, n .
\end{aligned}
$$

引理 9 记 $\left\{E_{j, i_{1}, i_{2}, i_{3}}\left[d\left(H_{1}, C_{01} \cup C_{02} \cup C\right)^{\perp}\right]\right\}$ 是 $d\left(H_{1}, C_{01} \cup C_{02} \cup C\right)^{\perp}$ 的四 元重量分布, 则

(i)

(ii)

$$
\begin{aligned}
& \sum_{j=1}^{t_{1}} E_{j, 0,0, i_{3}}\left[d\left(H_{1}, C_{01} \cup C_{02} \cup C\right)^{\perp}\right] \\
= & (s-1)\left[B_{i_{3}, 0}(d)-A_{i_{3}}\left(C_{1}\right)\right]+\left(\begin{array}{c}
n_{1} \\
i_{3}
\end{array}\right)(s-1)^{i_{3}},
\end{aligned}
$$

$$
\sum_{j=1}^{t_{1}} E_{j, 0,1, i_{3}}\left[d\left(H_{1}, C_{01} \cup C_{02} \cup C\right)^{\perp}\right]=(s-1) B_{i_{3}+1,1}(d),
$$

(iii)

$$
\sum_{j=1}^{t_{1}} E_{j, 1,0, i_{3}}\left[d\left(H_{1}, C_{01} \cup C_{02} \cup C\right)^{\perp}\right]=(s-1) B_{i_{3}+1,2}(d),
$$

(iv)

$$
\begin{aligned}
& \sum_{j=1}^{t_{1}} E_{j, 1,1, i_{3}}\left[d\left(H_{1}, C_{01} \cup C_{02} \cup C\right)^{\perp}\right] \\
= & (s-1)\left[B_{i_{3}, 3}(d)-A_{i_{3}+2,3}\left(C_{01} \cup C_{02} \cup C_{1}\right)\right]+\left(\begin{array}{c}
n_{1} \\
i_{3}
\end{array}\right)(s-1)^{i_{3}+1} \phi\left(P_{1}, C_{01}, C_{02}\right),
\end{aligned}
$$

其中 $l\left(P_{1}, C_{01}, C_{02}\right)=\#\left\{\right.$ 线性相关的三元组 $\left(a_{1}, a_{2}, a_{3}\right): a_{1} \in P_{1}, a_{2} \in C_{01}, a_{3} \in$ $\left.C_{02}\right\}$. 
引理 10 令 $\left\{E_{j, i_{1}, i_{2}, i_{3}}\left[d\left(H_{1}, C_{01} \cup C_{02} \cup C\right)^{\perp}\right]\right\}$ 是 $d\left(H_{1}, C_{01} \cup C_{02} \cup C\right)^{\perp}$ 的 四元重量分布，则有

$$
\begin{aligned}
& E_{j, i_{1}, i_{2}, i_{3}}\left[d\left(H_{1}, C_{01} \cup C_{02} \cup C\right)^{\perp}\right] \\
= & \gamma_{r_{1}}\left(i_{1}\right) \gamma_{r_{2}}\left(i_{2}\right) E_{j, 0,0, i_{3}}\left[d\left(H_{1}, C_{01} \cup C_{02} \cup C\right)^{\perp}\right] \\
& +\gamma_{r_{1}}\left(i_{1}\right) \alpha_{r_{2}}\left(i_{2}\right) E_{j, 0,1, i_{3}}\left[d\left(H_{1}, C_{01} \cup C_{02} \cup C\right)^{\perp}\right] \\
& +\alpha_{r_{1}}\left(i_{1}\right) \gamma_{r_{2}}\left(i_{2}\right) E_{j, 1,0, i_{3}}\left[d\left(H_{1}, C_{01} \cup C_{02} \cup C\right)^{\perp}\right] \\
& +\alpha_{r_{1}}\left(i_{1}\right) \alpha_{r_{2}}\left(i_{2}\right) E_{j, 1,1, i_{3}}\left[d\left(H_{1}, C_{01} \cup C_{02} \cup C\right)^{\perp}\right], \\
& \quad j=0, \cdots, t_{1}, \quad i_{1}=0, \cdots, L_{r_{1}}, \quad i_{2}=0, \cdots, L_{r_{2}}, \quad i_{3}=0, \cdots, n .
\end{aligned}
$$

总结上述引理 8 10, 可得如下两个定理:

定理 6 一个正规的 FFSP $s^{n_{1}-p_{1}} \cdot\left(s^{r_{1}}\right)\left(s^{r_{2}}\right) s^{n_{2}}$ 设计 $d=d\left(C_{1}, C_{01}, C_{02}, C_{2}\right)$ 与其参照设计 $d_{R}$ 的附加字长型 $\left\{B_{i, j}(d)\right\}$ 和 $\left\{B_{i, j}\left(d_{R}\right)\right\}$ 满足以下等式:

$$
\begin{aligned}
& B_{i, 0}(d)+\eta_{1} B_{i+1,1}(d)+\eta_{2} B_{i+1,2}(d)+\eta_{3} B_{i+2,3}(d) \\
= & c_{4}+\sum_{j=0}^{i} \theta_{i, j}^{(1)}\left[B_{i, 0}\left(d_{R}\right)+\eta_{1} B_{i+1,1}\left(d_{R}\right)+\eta_{2} B_{i+1,2}\left(d_{R}\right)+\eta_{3} B_{i+2,3}\left(d_{R}\right)\right] \\
& +\sum_{j=0}^{i-2}\left(\theta_{i, j}^{(2)}-\theta_{i, j}^{(1)}\right)\left[A_{j}\left(F_{1}\right)+\eta_{3} A_{j+2,3}\left(C_{01} \cup C_{02} \cup F_{1}\right)\right], \quad i=0, \cdots, n+2,
\end{aligned}
$$

其中 $\eta_{1}, \eta_{2}$ 和 $\eta_{3}$ 是只依赖于参数 $t, n, t_{1}, n_{1}, r_{1}, r_{2}$ 和 $s$ 的常数，常数 $c_{4}$ 还依赖 于 $P$ 中的不交的三元子平面 $\left(P_{1}, C_{01}, C_{02}\right)$ 的选取.

定理 $7\left\{B_{i, j}(d)\right\}$ 和 $\left\{B_{i, j}\left(d_{R}\right)\right\}$ 分别是一个正规的 FFSP $s^{n_{1}-p_{1}} \cdot\left(s^{r_{1}}\right)\left(s^{r_{2}}\right) s^{n_{2}}$ 设计 $d=d\left(C_{1}, C_{01}, C_{02}, C_{2}\right)$ 与其参照设计 $d_{R}$ 的附加字长型, 则有

$$
\begin{aligned}
B_{i, 0}(d)= & c_{5}+\sum_{k=0}^{i-2} \eta_{i, k}^{(1)} A_{k}\left(F_{1}\right)+(-1)^{i}\left[\sum_{j=0}^{3} B_{i, j}\left(d_{R}\right)-A_{i, 3}\left(C_{01} \cup C_{02} \cup F_{1}\right)\right] \\
& + \text { 低阶项, } \quad i=2, \ldots, n+1,
\end{aligned}
$$

其中 $c_{5}$ 和 $\eta_{i, j}^{(1)}$ 是常数, 且出现在上式右边的低阶项意指 $\left\{B_{k, j}\left(d_{R}\right), A_{k, 3}\left(C_{01} \cup\right.\right.$ $\left.\left.C_{02} \cup F_{1}\right)\right\}_{1 \leqslant k \leqslant i-1}$ 中的项.

\section{3 识别 MSA FFSP 设计的规则}

考虑正规的 $\operatorname{FFSP} s^{n_{1}-p_{1}} \cdot\left(s^{r_{2}}\right) s^{n_{2}}$ 设计 $d\left(C_{1}, C_{02}, C_{2}\right)$. 注意到

$$
B_{i, 0}\left[d\left(C_{1}, C_{02}, C_{2}\right)\right]=B_{i, 0}(d), \quad B_{i, 1}\left[d\left(C_{1}, C_{02}, C_{2}\right)\right]=B_{i, 1}(d),
$$

由定理 3 , 有

$$
\begin{aligned}
& B_{i, 0}(d)+\eta_{1} B_{i+1,1}(d) \\
= & c_{6}+\sum_{k=0}^{i-2} \eta_{i, k}^{(2)} A_{k}\left(F_{1}\right)+(-1)^{i}\left\{B_{i, 0}\left[d\left(F_{1}, C_{02}, C_{01} \cup F_{2}\right)\right]\right. \\
& \left.+\eta_{1} B_{i+1,1}\left[d\left(F_{1}, C_{02}, C_{01} \cup F_{2}\right)\right]\right\}+ \text { 低阶项, } \quad i=1, \cdots, n+1,
\end{aligned}
$$


其中 $c_{6}$ 和 $\eta_{i, k}^{(2)}$ 是常数.

应用等式

$$
\begin{aligned}
B_{i, 0}\left[d\left(F_{1}, C_{02}, C_{01} \cup F_{2}\right)\right] & =B_{i, 0}\left(d_{R}\right)+B_{i, 2}\left(d_{R}\right)+\text { 低阶项, } \\
B_{i+1,1}\left[d\left(F_{1}, C_{02}, C_{01} \cup F_{2}\right)\right] & =B_{i+1,1}\left(d_{R}\right)+B_{i+1,3}\left(d_{R}\right)+\text { 低阶项 }
\end{aligned}
$$

得到如下关系式:

$$
\begin{aligned}
& B_{i, 0}(d)+\eta_{1} B_{i+1,1}(d) \\
= & c_{7}+\sum_{k=0}^{i-2} \eta_{i, k}^{(2)} A_{k}\left(F_{1}\right)-.5 \mathrm{~cm}+(-1)^{i}\left[B_{i+1,1}\left(d_{R}\right)+B_{i+1,3}\left(d_{R}\right)\right]+\text { 低阶项. }
\end{aligned}
$$

再考虑正规 $\operatorname{FFSP} s^{n_{1}-p_{1}} \cdot\left(s^{r_{1}}\right) s^{n_{2}}$ 设计 $d\left(C_{1}, C_{01}, C_{2}\right)$, 可得到另一个关系式

$$
\begin{aligned}
& B_{i, 0}(d)+\eta_{2} B_{i+1,2}(d) \\
= & c_{8}+\sum_{k=0}^{i-2} \eta_{i, k}^{(3)} A_{k}\left(F_{1}\right)+(-1)^{i}\left[B_{i+1,2}\left(d_{R}\right)+B_{i+1,3}\left(d_{R}\right)\right]+\text { 低阶项, }
\end{aligned}
$$

其中 $c_{8}$ 和 $\eta_{i, k}^{(3)}$ 是常数.

综合定理 6 和 7 及 (15) 和 (16) 式, 可知依次最小化 $d$ 的附加字长型

$$
\left\{B_{2,0}(d), B_{2,1}(d), B_{2,2}(d), B_{2,3}(d), B_{3,0}(d), B_{3,1}(d), B_{3,2}(d), B_{3,3}(d)\right\}
$$

等价于依次最小化 $d_{R}$ 的如下成分:

$$
\begin{gathered}
\left\{\sum_{j=0}^{3} B_{2, j}\left(d_{R}\right),-\left[B_{2,1}\left(d_{R}\right)+B_{2,3}\left(d_{R}\right)\right],-\left[B_{2,2}\left(d_{R}\right)+B_{2,3}\left(d_{R}\right)\right],\right. \\
B_{2,3}\left(d_{R}\right),-\sum_{j=0}^{3} B_{3, j}\left(d_{R}\right)+A_{3,3}\left(C_{01} \cup C_{02} \cup F_{1}\right), \\
\left.B_{3,1}\left(d_{R}\right)+B_{3,3}\left(d_{R}\right), B_{3,2}\left(d_{R}\right)+B_{3,3}\left(d_{R}\right),-B_{3,3}\left(d_{R}\right)\right\} .
\end{gathered}
$$

因此, 结合文献 [9] 中的定理 6, 可以得到在 $P$ 中确定的三元不相交的子平 面下, 通过参照设计的字长型来识别 MSA 正规 FFSP 设计的一般规则:

定理 8 记 $\left\{A_{i, j}(d)\right\},\left\{B_{i, j}(d)\right\}$ 和 $\left\{A_{i, j}\left(d_{R}\right)\right\},\left\{B_{i, j}\left(d_{R}\right)\right\}$ 分别是一个正规 的 FFSP $s^{n_{1}-p_{1}} \cdot\left(s^{r_{1}}\right)\left(s^{r_{2}}\right) s^{n_{2}}$ 设计 $d=d\left(C_{1}, C_{01}, C_{02}, C_{2}\right)$ 与其参照设计 $d_{R}$ 的字 长型和附加字长型, 则 $d$ 在 $P$ 中确定的三元不相交的子平面 $\left(P_{1}, C_{01}, C_{02}\right)$ 下有 MSA, 当且仅当 $d_{R}$ 在同构的意义下是先依次最小化序列 (18)、然后依次最小化 序列 (17) 的唯一的设计.

$$
\begin{aligned}
& \left\{(-1)^{i} \sum_{j=0}^{3} A_{i, j}\left(d_{R}\right),(-1)^{i}\left[A_{i, 0}\left(d_{R}\right)+A_{i, 2}\left(d_{R}\right)\right],\right. \\
& \left.(-1)^{i}\left[A_{i, 0}\left(d_{R}\right)+A_{i, 1}\left(d_{R}\right)\right],(-1)^{i} A_{i, 0}\left(d_{R}\right)\right\}_{3 \leqslant i \leqslant f+2} .
\end{aligned}
$$

当 $r_{1}=r_{2}$ 时, 1 类束和 2 类束是同等重要的, 所以它们可以重新定义为 1 类 束. 相继地, 3 类束重新定义为 2 类束. 根据定理 8 的推导, 很容易证明下面的 
定理 9 记 $\left\{A_{i, j}(d)\right\},\left\{B_{i, j}(d)\right\}$ 和 $\left\{A_{i, j}\left(d_{R}\right)\right\},\left\{B_{i, j}\left(d_{R}\right)\right\}$ 分别是一个正规 的 FFSP $s^{n_{1}-p_{1}} \cdot\left(s^{r}\right)^{2} s^{n_{2}}$ 设计 $d=d\left(C_{1}, C_{01}, C_{02}, C_{2}\right)$ 与其参照设计 $d_{R}$ 的字长型 和附加字长型, 则 $d$ 在 $P$ 中确定的三元不相交的子平面 $\left(P_{1}, C_{01}, C_{02}\right)$ 下有 MSA, 当且仅当 $d_{R}$ 在同构意义下是先依次最小化序列 (19)、然后依次最小化序列 (20) 的唯一的设计.

$$
\begin{gathered}
\left\{(-1)^{i} \sum_{j=0}^{2} A_{i, j}\left(d_{R}\right),(-1)^{i}\left[2 A_{i, 0}\left(d_{R}\right)+A_{i, 1}\left(d_{R}\right)\right],(-1)^{i} A_{i, 0}\left(d_{R}\right)\right\}_{3 \leqslant i \leqslant f+2} . \\
\left\{\sum_{j=0}^{2} B_{2, j}\left(d_{R}\right),-\left[B_{2,1}\left(d_{R}\right)+2 B_{2,2}\left(d_{R}\right)\right], B_{2,3}\left(d_{R}\right),-\sum_{j=0}^{2} B_{3, j}\left(d_{R}\right)\right. \\
\left.+A_{3,3}\left(C_{01} \cup C_{02} \cup F_{1}\right), B_{3,1}\left(d_{R}\right)+2 B_{3,3}\left(d_{R}\right),-B_{3,3}\left(d_{R}\right)\right\} .
\end{gathered}
$$

由于 $P$ 中有多个非同构的三元不相交的子平面, 为了识别 MSA 正规 FFSP $s^{n_{1}-p_{1}} \cdot\left(s^{r_{1}}\right)\left(s^{r_{2}}\right) s^{n_{2}}$ 设计, 需要比较在每组非同构的三元不交的子平面下的 MSA FFSP 设计, 然后根据上述 MSA 准则选择出最优的设计.

\section{5 结束语}

本文得到了联系一个正规对称或混合水平 FFSP 设计与其参照设计的附加 字长型之间关系的一般而统一的组合等式，根据这些等式进一步建立了通过参 照设计来识别最小附加混杂对称或混合水平 FFSP 设计的一般而统一的规则. 正 如文献 [5,9] 中所说明的, 根据不同类的束的重要性的其他排序, 可以定义其他 类型的 MSA 混合水平 FFSP 设计, 本文中的规则也可以很方便地修改以适应它 们. 另外, 我们不难将这些方法和结果推广到包含多个高水平因子的更加一般 的情形.

\section{参考文 献}

1 Fries A, Hunter W G. Minimum aberration $2^{k-p}$ designs. Technometrics, 1980, 26: 225 232

2 Chen J, Sun D X, Wu C F J. A catalogue of two-level and three-level fractional factorial designs with small runs. Internat Statist Rev, 1993, 61(1): 131 145

3 Tang B, Wu C F J. Characterization of minimum aberration $2^{n-k}$ designs in terms of their complementary designs. Ann Statist, 1996, 25: 1176 1188

4 Suen C Y, Chen H, Wu C F J. Some identities on $q^{n-m}$ designs with application to minimum aberrations. Ann Statist, 1997, 25(3): 1176 1188

5 Wu C F J, Zhang R C. Minimum aberration designs with two-level and four-level factors. Biometrika, 1993, 80(1): 203 209

6 Wu C F J, Zhang R C, Wang R G. Construction of asymmetrical orthogonal arrays of the type $O A\left(s^{k}\right.$, $\left.\left(s^{r_{1}}\right)^{n_{1}} \ldots\left(s^{r_{t}}\right)^{n_{t}}\right)$. Statist Sinica, 1992, 2: 203 219

7 Zhang R C, Shao Q. Minimum aberration $\left(s^{2}\right) s^{n-k}$ designs. Statist Sinica, 2001, 11: 213 223

8 Mukerjee R, Wu C F J. Minimum aberration designs for mixed Factorials in terms of complementary sets. Statist Sinica, 2001, 11: 225 239 
9 Ai M Y, Zhang R C. Characterization of minimum aberration mixed factorials in terms of consulting designs. Statist Papers, 2004, 46(2): 157 171

10 Chen H, Cheng C S. Theory of optimal blocking of $2^{n-m}$ designs. Ann Statist, 1999, 27(6): 1948 1973

11 Zhang R C, Park D K. Optimal blocking of two-level fractional factorial designs. J Statist Plann Infer, 2000, 91: 107 121

12 Ai M Y, Zhang R C. Theory of minimum aberration blocked regular mixed factorial designs. J Statist Plann Infer, 2004, 126(1): 305 323

13 Ai M Y, Zhang R C. Theory of optimal blocking of nonregular factorial designs. Canad J Statist, 2004, 32(1): 57〜72

14 Box G E P, Jones S. Split-plot designs for robust product experimentation. J Appl Statist, 1992, 19: $3 \sim 26$

15 Bingham D, Sitter R R. Minimum aberration two-level fractional factorial split-plot designs. Technometrics, 1999, 41(1): $62 \sim 70$

16 Bingham D, Sitter R R. Some theoretical results for fractional factorial split-plot designs. Ann Statist, 1999, 27(4): 1240 1255

17 Ai M Y, Zhang R C. Multistratum fractional factorial split-plot designs with minimum aberration and maximum estimation capacity. Statist Probab Letters, 2004, 69(2): 161 170

18 艾明要, 何书元. 部分因析裂区设计最优分区组的理论. 中国科学, A 辑, 2005, 35(3): 265 272

19 Bingham D, Sitter R R. Design issues in fractional factorial split-plot experiments. J Quality Technology, 2001, 33(1): $2 \sim 15$

20 Mukerjee R, Fang K T. Fractional factorial split-plot designs with minimum aberration and maximum estimation capacity. Statist Sinica, 2002, 12: 885 903

21 MacWilliams T J, Sloane N J A. The Theory of Error-Correcting Codes. Amsterdam: North-Holland, 1977

22 Roman S. Coding and Information Theory. New York: Springer, 1992

23 Peterson W W, Weldon E J. Error-Correcting Codes. Berkeley: MIT Press, 1972

\section{附录}

引理 2 的证 由广义 MacWilliams 等式以及 Krawtchouk 多项式的性质可得

$$
\begin{aligned}
& E_{j_{1}, j_{2}}\left[d\left(H_{1}, C\right)^{\perp}\right] \\
= & s^{-t} \sum_{i_{1}=0}^{t_{1}} \sum_{i_{2}=0}^{n} P_{j_{1}}\left(i_{1} ; t_{1}\right) P_{j_{2}}\left(i_{2} ; n\right) E_{j_{1}, j_{2}}\left[d\left(H_{1}, C\right)\right] \\
= & s^{-t}\left[P_{j_{2}}(0 ; n)-P_{j_{2}}\left(s^{t-1} ; n\right)\right] P_{j_{1}}\left(0 ; t_{1}\right) \\
& +s^{-t} \sum_{i_{1}=0}^{t_{1}} \sum_{i_{2}=0}^{n} P_{j_{1}}\left(i_{1} ; t_{1}\right) P_{j_{2}}\left(i_{2} ; n\right) E_{i_{1}, s^{t-1}-i_{2}}\left[d\left(H_{1}, F\right)\right] \\
= & s^{-t}\left[P_{j_{2}}(0 ; n)-P_{j_{2}}\left(s^{t-1} ; n\right)\right] P_{j_{1}}\left(0 ; t_{1}\right) \\
& +s^{-t} \sum_{i_{1}=0}^{t_{1}} \sum_{i_{2}=s^{t-1}-n}^{s^{t-1}} P_{j_{1}}\left(i_{1} ; t_{1}\right) P_{j_{2}}\left(s^{t-1}-i_{2} ; n\right) E_{i_{1}, i_{2}}\left[d\left(H_{1}, F\right)\right] \\
= & s^{-t}\left[P_{j_{2}}(0 ; n)-P_{j_{2}}\left(s^{t-1} ; n\right)\right] P_{j_{1}}\left(0 ; t_{1}\right) \\
& +s^{-t_{1}-f} \sum_{k_{1}=0}^{t_{1}} \sum_{k_{2}=0}^{f}\left[\sum_{i_{1}=0}^{t_{1}} \sum_{i_{2}=s^{t-1}-n}^{s^{t-1}} P_{j_{1}}\left(i_{1} ; t_{1}\right) P_{j_{2}}\left(s^{t-1}-i_{2} ; n\right) P_{i_{1}}\left(k_{1} ; t_{1}\right) P_{i_{2}}\left(k_{2} ; f\right)\right] \\
& \cdot E_{k_{1}, k_{2}}\left[d\left(H_{1}, F\right)^{\perp}\right]
\end{aligned}
$$




$$
\begin{aligned}
= & s^{-t}\left[P_{j_{2}}(0 ; n)-P_{j_{2}}\left(s^{t-1} ; n\right)\right] \sum_{j_{1}=1}^{t_{1}} P_{j_{1}}\left(0 ; t_{1}\right) \\
& +s^{-f} \sum_{k_{1}=1}^{t_{1}} \sum_{k_{2}=0}^{f}\left[\sum_{i_{2}=s^{t-1}-n}^{s^{t-1}} P_{j_{2}}\left(s^{t-1}-i_{2} ; n\right) P_{i_{2}}\left(k_{2} ; f\right)\right] E_{k_{1}, k_{2}}\left[d\left(H_{1}, F\right)^{\perp}\right] \\
= & \theta_{j_{2}}^{(1)}+s^{-f} \sum_{k_{2}=0}^{f}\left[\sum_{i_{2}=0}^{f} P_{j_{2}}\left(s^{t-1}-i_{2} ; n\right) P_{i_{2}}\left(k_{2} ; f\right)\right] \sum_{k_{1}=1}^{t_{1}} E_{k_{1}, k_{2}}\left[d\left(H_{1}, F\right)^{\perp}\right] \\
= & \theta_{j_{2}}^{(1)}+\sum_{k_{2}=0}^{f} \theta_{j_{2}, k_{2}}^{(1)}\left\{\sum_{k_{1}=1}^{t_{1}} E_{k_{1}, k_{2}}\left[d\left(H_{1}, F\right)^{\perp}\right]\right\} .
\end{aligned}
$$

引理 3 的证 只需证明 (i), (ii) 的证明类似, 这里从略.

$\Omega(n)$ 为 $G F(s)$ 上 $n$ 维向量的集合. 对于 $i \geqslant 1$, 令 $\Omega(i, n)$ 为 $G F(s)$ 上有 $i$ 个非零元素的 $n$ 维向量的集合, 且

$$
\Omega^{*}(i, n)=\left\{\beta: \beta \in \Omega(i, n), \beta \text { 的最后 } n_{2} \text { 个元素非零 }\right\} .
$$

由于成比例的束是相同的, 根据 $A_{i}\left(C_{1}\right)$ 和 $B_{i}(d)$ 的定义, 得到

$$
A_{i}\left(C_{1}\right)=(s-1)^{-1} \#\left\{\beta_{1}: \beta_{1} \in \Omega\left(i, n_{1}\right), V\left(C_{1}\right) \beta_{1}=0\right\},
$$

且

$$
\begin{aligned}
B_{i}(d) & =(s-1)^{-1} \#\left\{\beta: \beta \in \Omega^{*}(i, n), V(C) \beta \text { 非零且与 } P_{1} \text { 中的某点成比例 }\right\} \\
& =(s-1)^{-1} \#\left\{(\alpha, \beta)^{\mathrm{T}}: \alpha \in \Omega\left(t_{1}\right), \alpha \neq 0, \beta \in \Omega^{*}(i, n), V(C) \beta+V\left(H_{1}\right) \alpha=0\right\},
\end{aligned}
$$

因此

$$
\begin{aligned}
& \sum_{j=1}^{t_{1}} E_{j, i}\left[d\left(H_{1}, C\right)^{\perp}\right] \\
= & \sum_{j=1}^{t_{1}} \#\left\{(\alpha, \beta)^{\mathrm{T}}: \alpha \in \Omega\left(j, t_{1}\right), \beta \in \Omega(i, n), V\left(H_{1}, C\right)(\alpha, \beta)^{\mathrm{T}}=0\right\} \\
= & \#\left\{(\alpha, \beta)^{\mathrm{T}}: \alpha \in \Omega\left(t_{1}\right), \alpha \neq 0, \beta \in \Omega(i, n), V\left(H_{1}, C\right)(\alpha, \beta)^{\mathrm{T}}=0\right\} \\
= & (s-1) B_{i}(d)+\#\left\{\left(\alpha, \beta_{1}\right)^{\mathrm{T}}: \alpha \in \Omega\left(t_{1}\right), \alpha \neq 0, \beta_{1} \in \Omega\left(i, n_{1}\right), V\left(H_{1}, C_{1}\right)\left(\alpha, \beta_{1}\right)^{\mathrm{T}}=0\right\} \\
= & (s-1) B_{i}(d)+\#\left\{\left(\alpha, \beta_{1}\right)^{\mathrm{T}}: \alpha \in \Omega\left(t_{1}\right), \beta_{1} \in \Omega\left(i, n_{1}\right), V\left(H_{1}, C_{1}\right)\left(\alpha, \beta_{1}\right)^{\mathrm{T}}=0\right\} \\
& -\#\left\{\beta_{1}: \beta_{1} \in \Omega\left(i, n_{1}\right), V\left(C_{1}\right) \beta_{1}=0\right\} \\
= & (s-1) B_{i}(d)+\left(\begin{array}{c}
n_{1} \\
i
\end{array}\right)(s-1)^{i}-(s-1) A_{i}\left(C_{1}\right) .
\end{aligned}
$$

定理 4 的证 由引理 3 和 6 及 7 可得

$$
\begin{aligned}
& (s-1)\left\{B_{i}\left[d\left(F_{1}, C_{0} \cup F_{2}\right)\right]-A_{i}\left(F_{1}\right)\right\}+\left(\begin{array}{c}
f_{1} \\
i
\end{array}\right)(s-1)^{i} \\
= & \sum_{j=1}^{t_{1}} E_{j, i}\left[d\left(H_{1},\left(C_{0} \cup F\right)\right)^{\perp}\right] \\
= & \sum_{j=1}^{t_{1}} \sum_{i_{1}+i_{2}=i} E_{j, i_{1}, i_{2}}\left[d\left(H_{1}, C_{0} \cup F\right)^{\perp}\right]
\end{aligned}
$$




$$
\begin{aligned}
= & \sum_{j=0}^{t_{1}} \sum_{i_{2}=0}^{i}\left\{\alpha_{r}\left(i-i_{2}\right) E_{j, 1, i_{2}}\left[d\left(H_{1}, C_{0} \cup F\right)^{\perp}\right]+\gamma_{r}\left(i-i_{2}\right) E_{j, 0, i_{2}}\left[d\left(H_{1}, C_{0} \cup F\right)^{\perp}\right]\right\} \\
= & (s-1) \sum_{i_{2}=0}^{i}\left\{\alpha_{r}\left(i-i_{2}\right) B_{i_{2}+1,1}\left(d_{R}\right)\right. \\
& \left.+\gamma_{r}\left(i-i_{2}\right)\left[B_{i_{2}, 0}\left(d_{R}\right)-A_{i_{2}}\left(F_{1}\right)+\left(\begin{array}{c}
f_{1} \\
i_{2}
\end{array}\right)(s-1)^{i_{2}-1}\right]\right\} .
\end{aligned}
$$

注意到

$$
\gamma_{r}(0)=1, \quad \gamma_{r}(1)=0, \quad \gamma_{r}(2)=0
$$

于是

$$
\begin{aligned}
& B_{i}\left[d\left(F_{1}, C_{0} \cup F_{2}\right)\right] \\
= & c_{8}+\sum_{i_{2}=0}^{i} \alpha_{r}\left(i-i_{2}\right) B_{i_{2}+1,1}\left(d_{R}\right)+\sum_{i_{2}=0}^{i} \gamma_{r}\left(i-i_{2}\right) B_{i_{2}, 0}\left(d_{R}\right)-\sum_{i_{2}=0}^{i-3} \gamma_{r}\left(i-i_{2}\right) A_{i_{2}}\left(F_{1}\right) .
\end{aligned}
$$

用上式替换定理 1 中的 $B_{i}\left(d_{R}\right)$ 可知定理 4 成立. 